\title{
Medidas de polarização da distribuição da renda e sua evolução no Brasil de 1995 a 2013 *
}

\author{
Rodolfo Hoffmann **
}

\begin{abstract}
Resumo
As principais medidas de polarização em uso são criticamente avaliadas. Posteriormente, medidas de polarização baseadas no índice de Gini ou no índice $T$ de Theil, considerando a divisão da distribuição da renda em dois ou três estratos, são aplicadas à distribuição da renda domiciliar per capita no Brasil, no período de 1995 a 2013. Constata-se clara tendência de redução da polarização, acompanhando a redução da desigualdade. Uma análise de dados regionais mostra o comportamento divergente da polarização da distribuição da renda no Distrito Federal. Finalmente, procura-se explicar os erros de trabalhos com resultados contrários.
\end{abstract}

Palavras-chave: Polarização; Distribuição da renda; Brasil - Desigualdade; Estratos de renda.

\section{Abstract \\ Polarization measures of income distribution in Brazil and their evolution from 1995 to 2013}

In this study the main measures of polarization in use are critically evaluated. Following this, polarization measures based on the Gini index or on Theil's $T$ inequality index, considering the division of the income distribution in two or three strata, are applied to data on per capita household income in Brazil, from 1995 to 2013. A clear tendency of decreasing income polarization is observed, similar to the reduction in inequality. Analyzing regional data, it is observed that the trend in income polarization is considerably different in the Federal District. Finally, explanations of the mistakes of papers with opposite results are presented.

Key words: Polarization; Income distribution; Brazil - Inequality; Income strata. JEL D31, D39, D63.

\section{Introdução}

O conceito e a medida da polarização de uma distribuição de renda ainda não estão bem estabelecidos. Há medidas de bipolarização e medidas mais amplas de multipolarização que, eventualmente, não são corretamente interpretadas. Quando se fala em "polarização" é usual pensar em dois polos (positivo e negativo, norte e sul). É razoável, então, considerar que uma maior polarização da distribuição de renda corresponda a uma maior densidade de probabilidade nas caudas, isto é, um crescimento nas frequências relativas de pobres e de ricos. O aumento da polarização está associado à redução da classe média.

* Artigo recebido em 9 de setembro de 2016 e aprovado em 30 de setembro de 2016.

** Professor Sênior da Escola Superior de Agricultura Luiz de Queiroz (Esalq), da Universidade de São Paulo (USP), Piracicaba, SP, Brasil. O autor agradece a Josimar Gonçalves de Jesus e a um parecerista de Economia e Sociedade pelas correções e valiosas sugestões para aperfeiçoar o artigo. E-mail: hoffmannr@usp.br. 
Um aumento da desigualdade da distribuição da renda significa, usualmente, um crescimento do número de pessoas relativamente pobres e/ou a concentração da renda nos mais ricos. À primeira vista, maior polarização se confunde com maior desigualdade. Há, realmente, autores que usam o termo "polarização" como um recurso retórico para ressaltar o fato de que maior desigualdade está associada a mais pobreza relativa e/ou mais concentração da renda a favor dos ricos. Mas, como será esclarecido na próxima seção, "polarização" pode ser definido como um conceito distinto de "desigualdade", podendo ocorrer aumento de polarização simultaneamente com redução da desigualdade. Para a análise científica é melhor que haja essa distinção entre os dois conceitos, pois isso possibilita aprofundar nossa compreensão das mudanças na distribuição da renda de um país.

De acordo com os dados da Pesquisa Nacional por Amostra de Domicílios (PNAD), de 1995 a 2014 ocorreu substancial redução da desigualdade da distribuição da renda no Brasil. Considerando, especificamente, a distribuição da renda domiciliar per capita, verifica-se que a redução da desigualdade foi mais intensa e sistemática de 2001 a 2014. Trata-se de um processo socioeconômico muito importante, que contribuiu para a redução da pobreza no País e gerou grande número de trabalhos avaliando sua intensidade e discutindo suas causas ${ }^{1}$. Veremos que alguns autores pretendem ter descoberto que em determinados períodos a redução da desigualdade foi acompanhada por aumento da polarização. Em princípio isso pode ocorrer, mas seria algo extraordinário, demandando uma explicação para um fenômeno tão inesperado. Veremos, entretanto, que esses autores calcularam medidas erradas e/ou não souberam interpretar seus resultados.

O objetivo principal deste artigo é, com base nos dados da PNAD, deixar claro como evoluiu a polarização da distribuição da renda domiciliar per capita no Brasil de 1995 a 2013. Para isso, na próxima seção, são analisadas, previamente, as medidas de polarização. Tendo em vista deixar mais claro o conceito de polarização e como ele se distingue do conceito de desigualdade, na seção 2 são comparadas as medidas de desigualdade e polarização para algumas distribuições teóricas. Na seção 3 os dados da PNAD são utilizados para mostrar como evoluiu a polarização da distribuição da RDPC no Brasil de 1995 a 2013 e na seção seguinte procura-se explicar por que existem artigos com resultados divergentes. A seção 5 mostra a evolução diferenciada da polarização em cinco regiões do Brasil, seguindo-se a conclusão do trabalho.

(1) Basta lembrar, por exemplo, o livro em dois volumes organizado por Barros, Foguel e Ulyssea (2006, 2007). 


\section{Medidas de polarização}

Como já foi comentado, o termo "polarização" pode se referir tanto a dois polos (norte e sul ou positivo e negativo) como a um número qualquer de polos (como ocorre, por exemplo, na análise de polos de desenvolvimento). Neste trabalho a palavra "polarização" é usada quando se considera o contraste entre 2 polos (ricos e pobres), tomando o cuidado de usar "multipolarização" quando o enfoque possibilita a existência de um número qualquer de polos, como acontece no caso da medida de Esteban e Ray (1994), discutida adiante.

A redução da classe média e o aumento da frequência relativa de pobres e ricos tende a aumentar a desigualdade da distribuição. Mas desigualdade e polarização de uma distribuição são conceitos distintos e podem até variar em sentidos opostos. Um exemplo numérico simples ajuda a entender isso. Considerese uma população com apenas 6 pessoas com rendas 1, 2, 3, 4, 5 e 6. Imagine que sejam feitas duas transferências progressivas de renda de uma unidade monetária: da $3^{\text {a }}$ para a $1^{\underline{a}}$ pessoa e da $6^{-}$para a $4^{\text {a }}$ pessoa. A nova distribuição tem 3 pessoas com renda 2 e 3 pessoas com renda 5. Uma vez que foram feitas duas transferências progressivas, a desigualdade diminuiu, mas a nova distribuição é mais polarizada. Note-se que a diferença entre a renda média da metade mais pobre (2) e a renda média da metade mais rica (5) continua a mesma, mas aumentou a identidade dentro dos dois polos. A ideia é que a polarização cresce com a separação (ou alienação) entre os polos e com a identidade dentro dos polos. Para o exemplo artificial descrito, o índice de Gini diminui de 0,278 para 0,214 e a medida de polarização de Wolfson, definida adiante [expressão (2)], aumenta de 0,151 para 0,214.

Em trabalho pioneiro, Foster e Wolfson (1992) criticam as tentativas anteriores de medir o declínio da classe média devido à necessidade de adotar valores muito arbitrários de renda para delimitar essa classe média. Eles propõem uma medida baseada na divisão em dois estratos com base na mediana. Essa medida foi reapresentada e analisada em dois artigos posteriores de Wolfson $(1994,1997)$, e vamos denominá-la medida de polarização de Wolfson $\left(P_{W}\right)$.

Em um gráfico da curva de Lorenz, denomina-se discrepância $(D)$ à diferença entre a ordenada da linha de perfeita igualdade e a ordenada da curva de Lorenz. Seja $D_{50}$ a discrepância no ponto correspondente à mediana, que é o segmento VT na Figura 1. Seja $\mu$ a renda média da população e sejam $\mu_{L}$ e $\mu_{U}$ as rendas médias para a metade mais pobre e para a metade mais rica. Foster e Wolfson (1992) definem a seguinte medida de distância relativa entre ricos e pobres ${ }^{2}$ :

(2) No original essa distância relativa é indicada por $T$, que substituímos por $\delta$ porque $T$ é reservado para representar uma das medidas de desigualdade de Theil. 


$$
\delta=\frac{\mu_{U}-\mu_{L}}{\mu}
$$

Como a renda média relativa de um estrato é igual à razão entre suas participações na renda e na população, temos

$$
\frac{\mu_{L}}{\mu}=\frac{0,5-D_{50}}{0,5} \quad \text { e } \quad \frac{\mu_{U}}{\mu}=\frac{0,5+D_{50}}{0,5}
$$

Subtraindo a primeira expressão da segunda, membro-a-membro, deduz-se que $\delta=$ $4 D_{50}$. Foster e Wolfson (1992) erram ao afirmar que $\delta$ é igual ao dobro de $D_{50}$.

Pode-se verificar que $D_{50}$ é igual ao índice de Gini da desigualdade entre os dois estratos, que indicaremos por $G_{e}$.

Na Figura 1, a linha SU é tangente à curva de Lorenz no ponto correspondente à mediana e pode-se verificar que a área do trapézio SOAU é igual a $D_{50}$. Então $\delta=4 D_{50}$ é igual a 4 vezes a área do trapézio SOAU, embora Foster e Wolfson (1992) afirmem que $\delta$ é o dobro dessa área. Levando em consideração esse engano, a sua proposta de medida de polarização deve ser escrita como

$$
P_{W}=\left(2 D_{50}-G\right) \frac{\mu}{m}=\left(2 G_{e}-G\right) \frac{\mu}{m}
$$

com $m$ indicando a mediana e $G$ representando o índice de Gini de toda a distribuição. A diferença $2 G_{e}-G$ é multiplicada pela medida de assimetria $\mu / m$.

A diferença $2 D_{50}-G$ corresponde, na Figura 1, ao dobro da área compreendida entre a tangente SU e a curva de Lorenz, mostrando que $P_{W} \geq 0$, com $P_{W}=0$ apenas se $G=0$.

A diferença $2 G_{e}-G$ pode ser interpretada como um contraste entre a desigualdade entre os dois estratos $\left(G_{e}\right)$ e a desigualdade dentro dos estratos, dada por $G-G_{e}$ :

$$
G_{e}-\left(G-G_{e}\right)=2 G_{e}-G
$$

As expressões (2) e (3) mostram que a medida de polarização de Wolfson cresce com a desigualdade entre as duas metades da distribuição $\left(G_{e}\right)$ e cresce, também, com a redução da desigualdade dentro das metades $\left(G-G_{e}\right)$. Em outras palavras, $P_{w}$ cresce com a separação (alienação) entre as duas metades e com a homogeneidade (identidade) dentro das metades.

Sendo $\Delta$ a diferença média da distribuição e lembrando que $G=\frac{\Delta}{2 \mu}$ e $\delta=$ $4 G_{e}$, temos

$$
2 G_{e}-G=\frac{\delta}{2}-\frac{\Delta}{2 \mu}=\frac{1}{2 \mu}\left(\mu_{U}-\mu_{L}-\Delta\right)
$$

mostrando que a medida de polarização está relacionada com a diferença relativa entre $\mu_{U}-\mu_{L}$ e $\Delta$. 
Figura 1

A curva de Lorenz e a sua tangente no ponto correspondente à mediana da distribuição

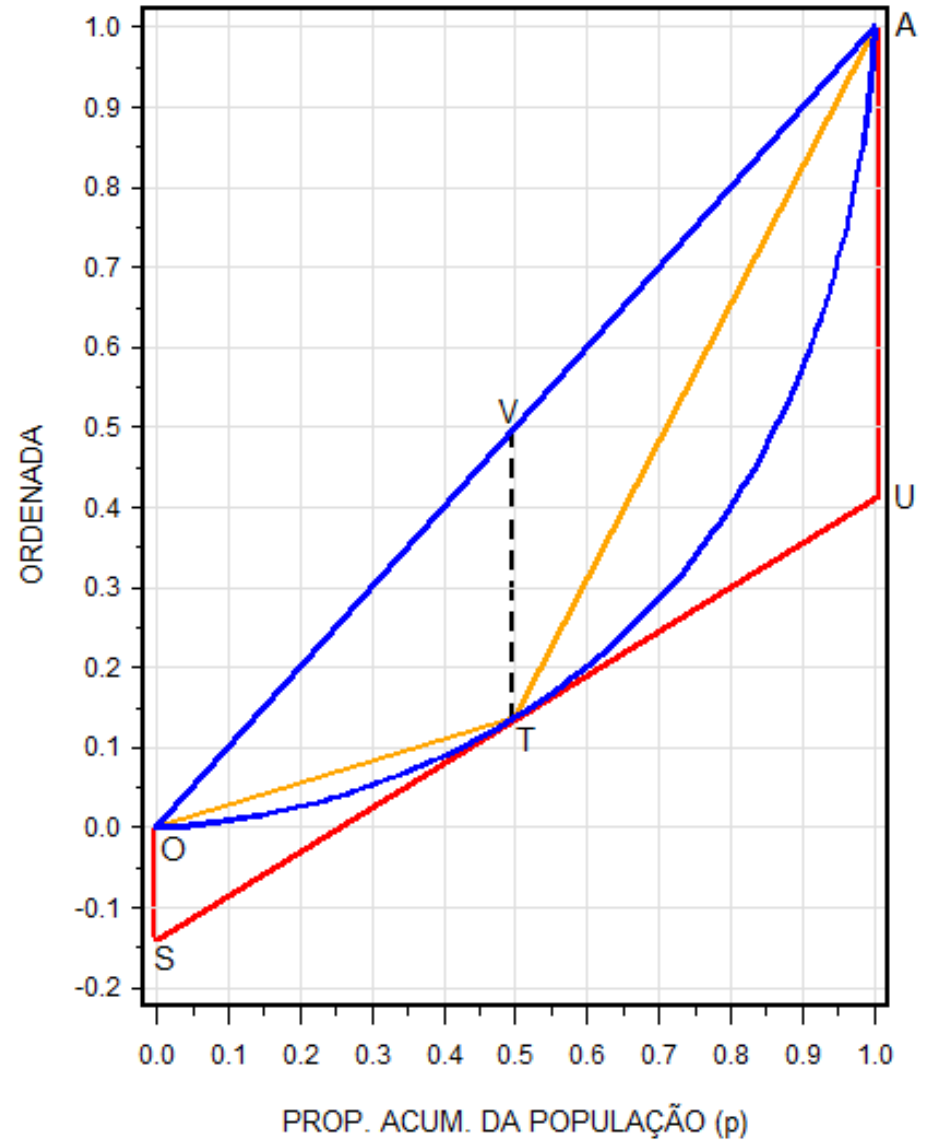

Wolfson (1994) modifica a fórmula da medida de polarização (2), multiplicando-a por 2. Como sua justificativa para isso é falha (ver Hoffmann, 2008), parece mais apropriado manter a fórmula (2).

Chakravarty (2009) faz uma exposição abrangente sobre o conceito e várias medidas de polarização. Ressalta que a distinção essencial entre os conceitos de polarização e de desigualdade é dada pelo axioma da bipolaridade aumentada, o qual estabelece que, quando uma distribuição de renda é dividida em duas metades (abaixo e acima da mediana), transferências progressivas envolvendo pessoas que estão e permanecem dentro de uma das metades causam aumento da polarização, ao mesmo tempo em que, de acordo com o princípio de Pigou-Dalton, reduzem a desigualdade dentro das metades e a desigualdade total, pois a desigualdade entre as duas metades permanece a mesma.

Curiosamente, Chakravarty (2009) apresenta, sem maior crítica, a medida de polarização de Milanovic (2000), que não obedece ao axioma da bipolaridade 
aumentada. A medida de Milanovic diminui quando é feita uma transferência progressiva entre os relativamente pobres e não é uma medida apropriada de polarização. Um exemplo numérico simples mostra o problema. Considere-se a distribuição de rendas definida pelo conjunto $A=\{1,3,5,7\}$. Após uma transferência progressiva entre as duas pessoas relativamente pobres as rendas são $B=\{2,2,5,7\}$. Em seguida, com uma transferência progressiva entre os dois relativamente ricos, $o$ conjunto fica $C=\{2,2,6,6\}$. A pretensa medida de polarização de Milanovic para os conjuntos $A, B$ e $C$ é, respectivamente, $0,5,0,475$ e 0,5 . A medida de Wolfson $\left(P_{W}\right)$, por sua vez, aumenta sucessivamente com as transferências progressivas que reduzem a desigualdade dentro das duas metades da distribuição, passando de 0,1875 no conjunto $A$ para 0,21875 no conjunto $B$, e atingindo 0,25 no conjunto $C$.

Com base na ideia de que uma medida de polarização da distribuição da renda é um contraste entre a desigualdade entre dois estratos e a desigualdade dentro dos dois estratos, podem ser definidas várias outras medidas, variando o limite entre estratos e a medida de desigualdade (Lopes, 2009). Quando o limite entre estratos não é a mediana, é necessário generalizar o axioma da bipolaridade aumentada, cujo enunciado passa a ser: transferências progressivas entre pessoas que estão e permanecem dentro de um dos dois estratos causam aumento da polarização, ao mesmo tempo em que, de acordo com o princípio de Pigou-Dalton, reduzem a desigualdade dentro dos estratos e a desigualdade total, pois a desigualdade entre os estratos permanece a mesma.

Dividindo a distribuição em dois estratos por meio da renda média e lembrando que a discrepância correspondente é a discrepância máxima $D_{M}$, podemos definir a medida de polarização

$$
P_{M}=2 D_{M}-G
$$

Curiosamente, a expressão (5) é um caso particular de uma medida muito mais geral, como mostram Esteban, Gradin e Ray (2007).

É interessante lembrar, nesse contexto, que a média é o limite entre dois estratos que maximiza o índice de Gini da desigualdade entre estratos (Aghevli; Mehran, 1981).

Pode-se verificar que a medida (5) também é igual a duas vezes a área compreendida entre a curva de Lorenz e uma tangente a essa curva no ponto correspondente à média. Como essa tangente à curva de Lorenz é paralela à linha de perfeita igualdade, o trapézio com área igual a $D_{M}$ é um paralelogramo. A medida $P_{M}$ é igual ao dobro da diferença entre a área desse paralelogramo e a área de desigualdade, podendo-se concluir que $P_{M}>0$ se $G>0$. Entretanto, com escolha arbitrária do limite entre os dois estratos e da própria medida de desigualdade, não há garantia de que o dobro da medida de desigualdade entre os dois estratos não seja inferior à medida geral de desigualdade, isto é, não há garantia de que a medida de 
polarização definida como um contraste entre a medida de desigualdade entre os dois estratos e a medida da desigualdade dentro dos estratos não possa ser negativa.

Adotando o índice $T$ de Theil como medida de desigualdade e considerando dois estratos delimitados pela média, podemos definir a medida de polarização

$$
P_{T M}=2 T_{e M}-T
$$

com $T_{e M}$ representando o valor do índice $T$ de Theil para a desigualdade entre esses dois estratos.

Se os dois estratos forem delimitados pelo $90^{\circ}$ percentil, temos

$$
P_{T 90}=2 T_{e 90}-T,
$$

com $T_{e 90}$ indicando o valor do $T$ de Theil relativo à desigualdade entre os $90 \%$ mais pobres e os $10 \%$ mais ricos.

Outra possibilidade seria considerar a divisão em dois estratos de maneira a maximizar o valor do $T$ de Theil referente à desigualdade entre os dois estratos.

Outra possibilidade, ainda, seria utilizar a divisão da distribuição entre "relativamente pobres" e "relativamente ricos" considerando o ponto no qual muda de sinal o efeito de um pequeno acréscimo na renda de uma pessoa sobre a medida de desigualdade (ver Hoffmann, 2001; Lambert; Lanza, 2006).

Como a ideia de polarização da distribuição de renda está associada ao aumento da frequência relativa de pobres e ricos, com redução da classe média, pode ser apropriado considerar a divisão da distribuição em 3 estratos. Evitando o uso de limites arbitrários, a divisão em 3 estratos pode ser feita de maneira a maximizar a desigualdade entre os 3 estratos, conforme a medida de desigualdade adotada. A metodologia básica para isso está descrita em Aghevli e Mehran (1981). Ver, também, Hoffmann (2007). Tanto para o índice de Gini como para as medidas de desigualdade $T$ e $L$ de Theil, a determinação dos dois limites exige um processo iterativo relativamente trabalhoso.

Seja $G_{e 3}$ o índice de Gini da desigualdade entre 3 estratos, delimitados de maneira a maximizar o valor dessa medida. Para as medidas de desigualdade de Theil ( $T$ e $L$ ) definimos, analogamente, $T_{e 3}$ e $L_{e 3}$. Podemos definir, então, três outras medidas de polarização como contrastes entre a desigualdade entre os 3 estratos e dentro dos 3 estratos:

$$
\begin{gathered}
P_{G 3}=2 G_{e 3}-G, \\
P_{T 3}=2 T_{e 3}-T
\end{gathered}
$$

e

$$
P_{L 3}=2 L_{e 3}-L
$$


Quando uma distribuição de renda é dividida em estratos, as três medidas de desigualdade mencionadas podem ser separadas em uma parcela referente à desigualdade entre estratos e uma parcela referente à desigualdade dentro dos estratos. No caso do índice $T$ de Theil essa última parcela é uma média ponderada dos valores de $T$ dentro de cada estrato, com ponderação pela participação do estrato na renda total. No caso do $L$ de Theil essa parcela também é uma média ponderada das medidas de desigualdade dentro de cada estrato, mas com ponderação pela participação de cada estrato na população. No caso do índice de Gini, entretanto, a parte do índice global referente à desigualdade dentro dos estratos é igual à soma ponderada dos índices de Gini dentro dos estratos, com fatores de ponderação iguais ao produto da participação de cada estrato na população e na renda total. A soma de tais fatores é, obviamente, menor do que 1, contribuindo para tornar relativamente menor a parcela do índice de Gini referente à desigualdade dentro dos estratos. Isso deve ser levado em consideração ao interpretar os resultados.

Esteban e Ray (1994) criaram uma medida para a multipolarização de uma distribuição de renda. Nesse caso pode haver apenas um ou muitos polos, de maneira análoga ao que acontece com os "polos de desenvolvimento econômico". A medida é rediscutida em Duclos, Esteban e Ray (2004) e Esteban, Gradín e Ray (2007). Ela capta a existência de conjuntos de pessoas com rendas semelhantes que se destacam dos demais membros da população. Os próprios autores perceberam o perigo do seu uso inapropriado e no artigo de 2007 afirmam que a medida só deve ser usada "after the population has been regrouped in a way that captures the group identification structure of society" (Esteban; Gradín; Ray, 2007, p. 4). Veremos adiante que essa medida tem sido erroneamente interpretada em estudos sobre a distribuição da renda no Brasil.

Handcock e Morris (1999), em livro sobre "Relative distribution methods in the social sciences", definem o que eles denominam de median relative polarization index (MRP). Trata-se de tentativa de captar se há ou não aumento de polarização por meio de comparação de duas distribuições: uma distribuição básica $A$ e uma outra distribuição $C$. Para isso deve-se construir uma distribuição intermediária $B$, deslocando a distribuição $A$ de maneira que fique com mediana igual à de $C$. Seja $R$ a posição de ordem, na distribuição $B$, dos quantis da distribuição $C$. Sendo $E$ o operador de esperança matemática, o índice MRP é definido como

$$
M R P=4 E(|R-0,5|)-1
$$

No caso de termos $n$ valores igualmente prováveis na distribuição final,

$$
M R P=\frac{4}{n} \sum_{i=1}^{n}\left|R_{i}-0,5\right|-1
$$

Como no cálculo do MRP somam-se os valores absolutos de $R_{i}-0,5$ acima e abaixo da mediana, verifica-se que ele não é afetado por transferências progressivas que envolvem pessoas que estão e permanecem abaixo da mediana ou por transferência progressivas entre pessoas que estão e permanecem acima da mediana. 
O índice MRP não obedece ao axioma da bipolaridade aumentada. Na realidade, a medida MRP avalia se na distribuição $C$ a desigualdade entre as duas metades é ou não maior do que na distribuição $B$. A leitura do livro de Handcock e Morris deixa claro que eles se referem a polarização como sinônimo de desigualdade.

O pior, ainda, é que os autores não deixam claro que o deslocamento de $A$ para $B$ deve ser feito por meio de alteração na escala, isto é, multiplicando todas as rendas por uma constante ou, equivalentemente, somando uma constante aos logaritmos das rendas. Apenas dessa maneira se evita que haja alteração da desigualdade (e da polarização) na passagem de $A$ para $B$. O livro dá a entender que o deslocamento de $A$ para $B$ pode ser feito adicionando uma constante a todas as rendas; se essa constante for positiva, haverá redução da desigualdade e a comparação de $C \operatorname{com} B$ poderá indicar mudanças na desigualdade com sinal oposto ao da diferença de desigualdade entre $C$ e $A$. Adiante veremos que esse é o erro cometido por Clementi e Schettino (2013) ao analisarem a evolução da polarização da distribuição da renda no Brasil de 2001 a 2011.

\section{Desigualdade e polarização em algumas distribuições teóricas}

Não há dúvida de que o conceito de qualquer fenômeno deve ser estabelecido antes de se definir uma medida de sua intensidade. Mas é inegável, também, que o uso de determinadas medidas ajuda a estabelecer o conceito. Nesta seção analisa-se o comportamento das medidas de polarização $P_{W}$ e $P_{M}$ para algumas distribuições teóricas, visando tornar mais claro o próprio conceito de polarização.

Todas as medidas usuais de desigualdade da distribuição de renda (índice de Gini, as medidas $T$ e $L$ de Theil, o coeficiente de variação, a porcentagem da renda apropriada pelo décimo mais rico) são insensíveis à unidade monetária usada. É claro que a desigualdade da distribuição da renda no Brasil não mudou, de um dia para o outro, nas diversas ocasiões em que a unidade monetária se tornou mil vezes maior. É razoável estabelecer que a polarização de uma distribuição também não é alterada quando todos os valores são multiplicados por uma constante.

Para ilustrar a questão, considere-se uma distribuição uniforme de $a$ a $b=a$ $+\theta, \operatorname{com} \theta>0$ e $a \geq 0$. A média e a mediana são iguais a $(a+b) / 2$ e pode-se demonstrar que o índice de Gini é igual a

$$
G=\frac{1}{3\left(2 \frac{a}{\theta}+1\right)}
$$

Como o valor de $G$ depende apenas da relação $a / \theta$, é óbvio que ele não é alterado com uma mudança de escala (equivalente a multiplicar todas as rendas por uma constante). Note-se que para $a=0$ o índice de Gini é igual a $1 / 3$, qualquer que seja o valor de $\theta$. Note-se, também, que um aumento no valor de $a$, que corresponde a uma translação da distribuição para a direita, causa redução do índice de Gini. 
Pode-se demonstrar que em uma distribuição uniforme de $a$ a $b=a+\theta$, a discrepância máxima, igual ao índice de Gini da desigualdade entre as duas metades, é

$$
D_{50}=\frac{1}{4\left(2 \frac{a}{\theta}+1\right)}
$$

e que o índice de polarização de Wolfson é

$$
P_{W}=\frac{1}{6\left(2 \frac{a}{\theta}+1\right)}=\frac{G}{2}
$$

Se $a=0, G=1 / 3$ e $P_{W}=1 / 6$. Aumento no valor de $a$ causa redução na desigualdade e na polarização, mantendo a relação $G=2 P_{W}$. Quando se comparam distribuições uniformes, a desigualdade e a polarização variam, necessariamente, no mesmo sentido.

No caso de uma distribuição uniforme, tanto o índice de Gini como a medida de polarização de Wolfson dependem apenas da relação $a / \theta$, não sendo afetados por uma mudança de escala que altere $a$ e $\theta$ na mesma proporção. Por outro lado, as duas medidas são reduzidas com uma translação da distribuição para a direita, isto é, um aumento no valor de $a$ sem alteração no valor de $\theta$. Ter essas propriedades em mente ajuda a entender a análise crítica da medida de polarização de Handcock e Morris feita anteriormente e que será usada posteriormente na crítica do artigo de Clementi e Schettino (2013).

No caso de uma distribuição exponencial, com função de densidade

$$
f(x)=\theta e^{-\theta x} \quad \text { para } x \geq 0,
$$

verifica-se que a média é $1 / \theta, G=0,5, \quad P_{W}=1-\frac{1}{2 \ln 2}$ e $\quad P_{M}=\frac{2}{e}-\frac{1}{2}$

Note-se que os valores de $G$ e das duas medidas de polarização não dependem do parâmetro $\theta$. Como nessa distribuição uma alteração em $\theta$ corresponde a uma mudança na unidade de medida de $x$, reafirma-se a ideia de que esse tipo de mudança não afeta a desigualdade nem a polarização.

Considere-se, finalmente, uma variável $x$ com distribuição log-normal, de maneira que o logaritmo neperiano da variável tem distribuição normal com média $\mu$ e variância $\sigma^{2}$. De acordo com Aitchison e Brown (1957), a média, a mediana e a moda de $x$ são $\exp \left(\mu+\frac{\sigma^{2}}{2}\right), \exp (\mu)$ e $\exp \left(\mu-\sigma^{2}\right)$, respectivamente, e o índice de Gini é

$$
G=2 \Phi\left(\frac{\sigma}{\sqrt{2}}\right)-1
$$

com $\Phi$ indicando a função de distribuição normal reduzida. Pode-se verificar que os índices de Gini da desigualdade entre as duas metades (acima e abaixo da mediana) ou entre as duas partes delimitadas pela média também dependem apenas de $\sigma$ e que no intervalo $0,25 \leq G \leq 0,75$ as medidas $P_{W}$ e $P_{M}$ são funções crescentes e aproximadamente lineares de $G$, como mostra a Figura 2. 
Figura 2

As medidas de polarização $P_{W}$ e $P_{M}$ em função do índice de Gini para uma distribuição log-normal

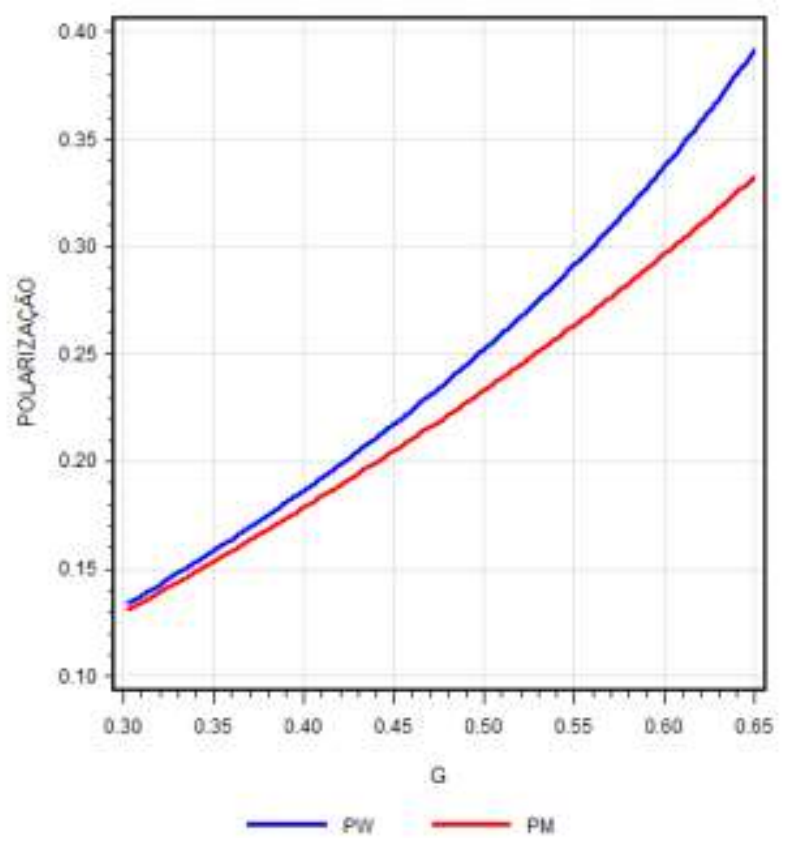

Multiplicar os valores de $x$ por uma constante, no caso de uma distribuição log-normal, altera apenas o parâmetro $\mu$, e não o parâmetro $\sigma^{2}$. Desnecessário dizer que, se uma constante for adicionada a todos os valores de $x$, a distribuição deixa de ser log-normal.

O comportamento das medidas $P_{W}$ e $P_{M}$ ilustrado na Figura 2 é relevante na medida em que a distribuição da renda é e permanece aproximadamente log-normal.

\section{Evolução da polarização da distribuição da RDPC no Brasil de 1995 a 2013}

Os dados da PNAD são usados para analisar a distribuição da RDPC no Brasil de 1995 a 2013. Como até 2003 a PNAD não cobria a área rural da antiga região Norte, sempre que a análise envolve comparações com resultados anteriores a 2004, os registros referentes a essa área foram excluídos dos arquivos de dados de 2004 a 2013. Todos os cálculos foram feitos usando a última versão dos fatores de ponderação fornecidos pelo IBGE.

Os resultados estão nas Tabelas 1 e 2 e a evolução das medidas de polarização de 1995 a 2013 pode ser mais facilmente visualizada nas Figuras 3 e 4 . Uma vez que os dados incluem domicílios cuja renda declarada é nula, não é possível calcular o índice $L$ de Theil para toda a distribuição e, consequentemente, não é possível calcular a medida de polarização dada pela expressão (10). 
Tabela 1

Evolução, de 1995 a 2013, do índice de Gini $(G)$, da discrepância máxima $\left(D_{M}\right)$ e de medidas de bipolarização da distribuição da RDPC no Brasil

\begin{tabular}{l|c|c|c|c|c|c|c}
\hline Ano & $G$ & $P_{W}$ & $P_{M}$ & $D_{M}$ & $100 \frac{D_{M}}{G}$ & $P_{T M}$ & $P_{T 90}$ \\
\hline 1995 & 0,599 & 0,312 & 0,296 & 0,447 & 74,7 & 0,149 & 0,193 \\
1996 & 0,600 & 0,318 & 0,296 & 0,448 & 74,6 & 0,146 & 0,181 \\
1997 & 0,600 & 0,316 & 0,295 & 0,448 & 74,6 & 0,140 & 0,181 \\
1998 & 0,598 & 0,305 & 0,294 & 0,446 & 74,6 & 0,142 & 0,190 \\
1999 & 0,592 & 0,298 & 0,291 & 0,442 & 74,6 & 0,145 & 0,191 \\
2001 & 0,594 & 0,302 & 0,290 & 0,442 & 74,4 & 0,129 & 0,182 \\
2002 & 0,587 & 0,295 & 0,287 & 0,437 & 74,4 & 0,129 & 0,182 \\
2003 & 0,581 & 0,289 & 0,281 & 0,431 & 74,2 & 0,125 & 0,173 \\
2004 & 0,569 & 0,279 & 0,273 & 0,421 & 74,0 & 0,111 & 0,156 \\
2005 & 0,566 & 0,273 & 0,270 & 0,418 & 73,8 & 0,106 & 0,162 \\
2006 & 0,560 & 0,265 & 0,266 & 0,413 & 73,7 & 0,103 & 0,156 \\
2007 & 0,552 & 0,261 & 0,257 & 0,405 & 73,3 & 0,094 & 0,140 \\
2008 & 0,543 & 0,251 & 0,252 & 0,397 & 73,2 & 0,085 & 0,132 \\
2009 & 0,539 & 0,246 & 0,248 & 0,393 & 73,0 & 0,078 & 0,125 \\
2011 & 0,527 & 0,232 & 0,238 & 0,382 & 72,6 & 0,069 & 0,117 \\
2012 & 0,524 & 0,228 & 0,236 & 0,380 & 72,5 & 0,043 & 0,098 \\
2013 & 0,523 & 0,226 & 0,234 & 0,379 & 72,4 & 0,064 & 0,115 \\
\hline
\end{tabular}

Tabela 2

Evolução, de 1995 a 2013, da desigualdade entre 3 estratos e respectivas medidas de polarização da distribuição da RDPC no Brasil

\begin{tabular}{ccc|c|c|c|c|c|c}
\hline Ano & $G_{e 3}$ & $100 \frac{G_{e 3}}{G}$ & $P_{G 3}$ & $T_{e 3}$ & $100 \frac{T_{e 3}}{T}$ & $P_{T 3}$ & $L_{e 3}$ \\
\hline 1995 & 0,530 & 88,6 & 0,462 & 0,589 & 81,0 & 0,450 & 0,556 \\
1996 & 0,531 & 88,5 & 0,462 & 0,587 & 80,9 & 0,448 & 0,565 \\
1997 & 0,531 & 88,5 & 0,463 & 0,589 & 80,6 & 0,447 & 0,563 \\
1998 & 0,530 & 88,6 & 0,462 & 0,589 & 80,9 & 0,449 & 0,556 \\
1999 & 0,524 & 88,5 & 0,456 & 0,574 & 81,3 & 0,441 & 0,541 \\
2001 & 0,525 & 88,4 & 0,456 & 0,579 & 80,5 & 0,438 & 0,544 \\
2002 & 0,520 & 88,5 & 0,452 & 0,567 & 80,5 & 0,429 & 0,528 \\
2003 & 0,513 & 88,4 & 0,446 & 0,549 & 80,7 & 0,418 & 0,515 \\
2004 & 0,502 & 88,3 & 0,435 & 0,525 & 80,0 & 0,394 & 0,488 \\
2005 & 0,500 & 88,3 & 0,434 & 0,522 & 80,2 & 0,394 & 0,481 \\
2006 & 0,493 & 88,2 & 0,427 & 0,509 & 80,2 & 0,383 & 0,467 \\
2007 & 0,486 & 88,0 & 0,420 & 0,488 & 79,5 & 0,362 & 0,454 \\
2008 & 0,477 & 87,9 & 0,412 & 0,470 & 79,3 & 0,347 & 0,436 \\
2009 & 0,473 & 87,9 & 0,408 & 0,463 & 78,8 & 0,339 & 0,428 \\
2011 & 0,462 & 87,7 & 0,397 & 0,442 & 78,9 & 0,323 & 0,407 \\
2012 & 0,459 & 87,6 & 0,394 & 0,441 & 76,4 & 0,305 & 0,400 \\
2013 & 0,458 & 87,6 & 0,393 & 0,434 & 78,6 & 0,316 & 0,400 \\
\hline
\end{tabular}


Como a discrepância máxima $D_{M}$ é igual ao índice de Gini entre os dois estratos correspondentes, verifica-se, na Tabela 1 , que a desigualdade entre apenas dois estratos representa quase $3 / 4$ do índice de Gini total. Na Tabela 2, considerando a divisão em 3 estratos, observa-se que a participação da desigualdade entre estratos no índice de Gini cai de 88,6\% em 1995 para 87,6\% em 2013. Para o $T$ de Theil essa participação também diminui, mantendo-se quase sempre acima de $78 \%$.

Davies e Shorrocks (1989) consideram surpreendente que a divisão da distribuição da renda no Canadá em 1983 em apenas 5 estratos já produzisse um índice de Gini entre estratos que representa $95 \%$ do índice de Gini total. Utilizando dados de RDPC da PNAD de 2013 para todo o Brasil, verifica-se que as divisões em 2, 3 ou 5 estratos produzem índices de Gini entre estratos que captam, respectivamente, $72,4 \%, 87,6 \%$ e $95,4 \%$ do total $(G=0,525)$. Considerando a divisão que maximiza o $T$ de Theil entre estratos, as porcentagens correspondentes são $60,8 \%, 78,6 \%$ e $90,8 \%$ (com $T=0,556$ ). Verifica-se, portanto, que a desigualdade entre um pequeno número de estratos representa grande parte da desigualdade da distribuição da RDPC no Brasil. Cabe ressaltar que a maior subdeclaração da renda dos mais ricos na PNAD deve contribuir para superestimar a participação da desigualdade entre estratos na desigualdade total.

Figura 3

Evolução de medidas de polarização baseadas na divisão da população em 2 estratos.

Brasil, 1995-2013

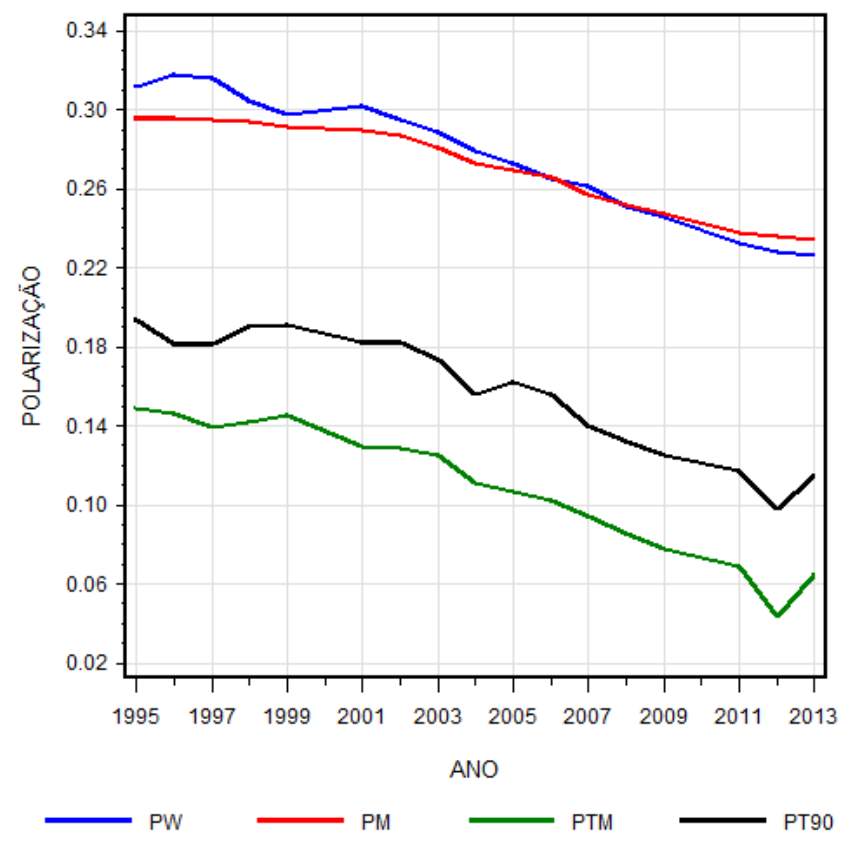


O exame das Tabelas 1 e 2 e das Figuras 3 e 4 mostra que ocorre clara redução da polarização no período analisado, acompanhando a redução da desigualdade ${ }^{3}$. Todas as medidas de polarização mostram tendência de decréscimo fortemente significativa. A correlação entre $P_{W}$ e $G$, por exemplo, é igual a 0,995 , com probabilidade caudal inferior a $0,01 \%$.

As três medidas de desigualdade entre três estratos apresentadas na Tabela $2\left(G_{e 3}, T_{e 3}\right.$ e $\left.L_{e 3}\right)$ mostram tendências decrescentes similares. As três mostram correlação negativa e fortemente significativa com a variável "ano" (correlações negativas com valor absoluto superior a 0,97 , com probabilidade caudal do teste de nulidade inferior a $0,01 \%$ ). Essas 3 medidas mostram correlações fortíssimas (maiores do que 0,99 ) entre si e com o índice de Gini e o $T$ de Theil referentes à desigualdade total.

Figura 4

Evolução das medidas de polarização baseadas na divisão da população em 3 estratos.

Brasil, 1995-2013

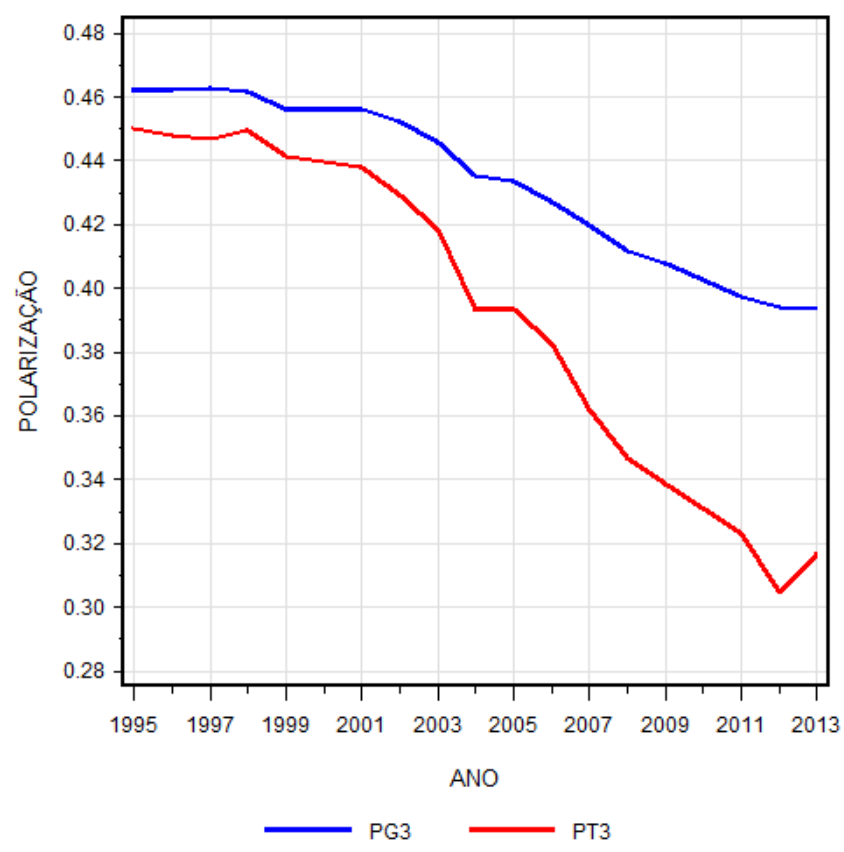

(3) Os dados da PNAD de 2014 mostram nova redução na desigualdade e das medidas de polarização $P_{W}$ e $P_{M}$. Em uma nova linha da Tabela 1, para 2014, os valores são: $G=0,513, P_{W}=0,220, P_{M}=0,231, D_{M}=$ 0,372 , correspondendo a $72,5 \%$ do índice de Gini, $P_{T M}=0,065$ e $P_{T 90}=0,113$. 
A Tabela 3 mostra os limites entre estratos que maximizam o índice de Gini entre 3 estratos de RDPC e as porcentagens da população e da renda em cada um dos 3 estratos.

Verifica-se que há tendências estatisticamente significativas (probabilidade caudal inferior a $0,01 \%$ ) de

a) decréscimo da porcentagem da população no $1^{\circ}$ estrato e crescimento das porcentagens no $2^{\circ}$ e no $3^{\circ}$ estratos,

b) crescimento da porcentagem da renda no $1^{\mathrm{o}}$ e no $2^{\mathrm{o}}$ estratos e decréscimo dessa porcentagem no 3 o estrato.

\section{Tabela 3}

Valor corrente dos limites entre os 3 estratos de RDPC, de maneira a maximizar o índice de Gini entre estratos, porcentagens da população e da renda em cada um dos 3 estratos e coeficiente para inflacionar os valores monetários para Reais de setembro-outubro de 2013

\begin{tabular}{|c|c|c|c|c|c|c|c|c|c|}
\hline \multirow[t]{2}{*}{ Ano } & \multirow{2}{*}{$\begin{array}{c}1^{\mathrm{o}} \\
\text { limite }\end{array}$} & \multirow{2}{*}{$\begin{array}{c}2^{\underline{o}} \\
\text { limite }\end{array}$} & \multicolumn{3}{|c|}{$\begin{array}{c}\% \text { da população no } \\
\text { estrato }\end{array}$} & \multicolumn{3}{|c|}{$\%$ da renda no estrato } & \multirow{2}{*}{$\begin{array}{c}\text { Coeficiente } \\
\text { para } \\
\text { inflacionar } \\
\text { in }\end{array}$} \\
\hline & & & 10 & $2 \underline{0}$ & 30 & $1 \underline{0}$ & $2 \underline{0}$ & $3 \underline{0}$ & \\
\hline 1995 & 354,7 & 1209,2 & 53,6 & 33,9 & 12,4 & 14,3 & 33,2 & 52,5 & 3,195132 \\
\hline 1996 & 361,8 & 1237,5 & 53,8 & 33,6 & 12,5 & 14,2 & 33,4 & 52,4 & 2,842479 \\
\hline 1997 & 362,3 & 1236,0 & 53,9 & 33,8 & 12,4 & 14,2 & 33,6 & 52,2 & 2,724354 \\
\hline 1998 & 364,3 & 1247,4 & 54,0 & 33,6 & 12,4 & 14,5 & 33,0 & 52,5 & 2,643176 \\
\hline 1999 & 346,0 & 1163,7 & 53,6 & 33,9 & 12,6 & 14,7 & 33,1 & 52,3 & 2,483367 \\
\hline 2001 & 350,5 & 1162,4 & 52,4 & 35,0 & 12,7 & 14,0 & 33,6 & 52,4 & 2,162740 \\
\hline 2002 & 352,5 & 1154,8 & 52,2 & 35,0 & 12,8 & 14,2 & 33,5 & 52,2 & 1,967530 \\
\hline 2003 & 335,2 & 1070,3 & 51,3 & 35,6 & 13,0 & 14,1 & 34,1 & 51,9 & 1,684117 \\
\hline 2004 & 353,4 & 1099,4 & 51,5 & 35,4 & 13,1 & 14,9 & 34,2 & 51,0 & 1,591221 \\
\hline 2005 & 380,2 & 1174,5 & 52,1 & 35,3 & 12,6 & 15,4 & 34,6 & 50,0 & 1,512446 \\
\hline 2006 & 418,2 & 1267,6 & 51,5 & 35,6 & 12,8 & 15,5 & 34,6 & 49,9 & 1,471428 \\
\hline 2007 & 433,7 & 1276,9 & 50,5 & 36,3 & 13,2 & 15,3 & 35,3 & 49,5 & 1,403391 \\
\hline 2008 & 462,5 & 1324,6 & 50,3 & 36,7 & 13,1 & 15,7 & 35,8 & 48,5 & 1,309748 \\
\hline 2009 & 475,8 & 1347,9 & 49,8 & 36,9 & 13,3 & 15,6 & 35,8 & 48,6 & 1,255619 \\
\hline 2011 & 515,8 & 1405,8 & 48,9 & 37,8 & 13,4 & 15,7 & 36,7 & 47,6 & 1,117431 \\
\hline 2012 & 557,2 & 1509,4 & 48,9 & 37,8 & 13,3 & 16,0 & 36,6 & 47,4 & 1,056364 \\
\hline 2013 & 577,7 & 1552,9 & 48,4 & 38,2 & 13,4 & 15,7 & 36,9 & 47,4 & 1,000000 \\
\hline
\end{tabular}

(1) Com base no INPC, transforma valores dos demais anos em Reais de setembro-outubro de 2013 , conforme procedimento proposto por Corseuil e Foguel (2002).

A Figura 5 mostra a evolução das participações de cada um dos 3 estratos na população e na renda total. Observa-se crescimento tanto da fração da população como da porcentagem da renda total que pertence ao estrato intermediário. Se esse estrato intermediário em uma divisão em 3 estratos que maximiza o índice de Gini entre estratos for identificado como a "classe média", os dados da PNAD revelam claro crescimento dessa classe média no período analisado. 
Figura 5

Evolução, de 1995 a 2013, das porcentagens da população (linha contínua) e da renda (linha tracejada) em três estratos da distribuição da RDPC no Brasil, delimitados de maneira a maximizar o índice de Gini entre estratos

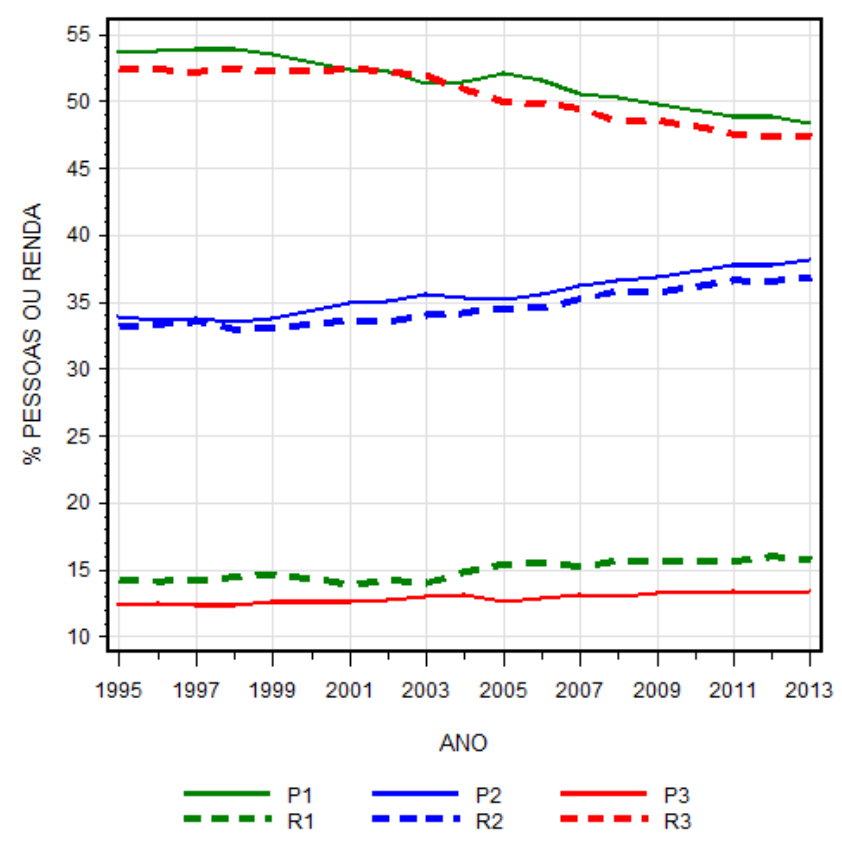

\section{Resultados convergentes e afirmativas divergentes na literatura sobre polarização da distribuição da renda no Brasil}

Scorzafave e Castro (2007) fizeram um trabalho pioneiro e competente sobre o cálculo de medidas de polarização da distribuição da renda no Brasil ${ }^{4}$. Já usam a divisão da distribuição em 2 ou 3 grupos. Obtêm "níveis de polarização que se mostraram alinhados com a sensível redução do índice de Gini verificada entre 1981 e 2003" e concluem, corretamente, que "o tão comentado fenômeno de desaparecimento da classe média não vem ocorrendo no Brasil".

No mesmo ano Figueirêdo, Netto Jr. e Porto Jr. Publicam um artigo no qual analisam dados de 1987 a 2003 e concluem que ocorreu "forte aumento" da polarização e "esvaziamento das classes intermediárias". Essas conclusões estão erradas, pois se fundamentaram em uma interpretação incorreta do índice de Polarização de Duclos, Esteban e Ray (2004), que capta todos os "polos" nos quais se agrupam rendas iguais, e não apenas "pobres" e "ricos". Hoffmann (2008) mostra que medidas de bipolarização da distribuição da renda no Brasil apresentam clara tendência geral decrescente de 1987 a 2005 e que a medida calculada por Figueirêdo

(4) Ver, também, Castro e Scorzafave (2005) e Castro (2006). 
et al. (2007) capta a multipolarização em picos de maior frequência de valores declarados associados ao salário mínimo e à tendência de as pessoas declararem números redondos 5 .

Audibert (2011) analisa a evolução da polarização da distribuição da renda no Brasil e no Rio Grande do Sul, de 2005 a 2009. Nos dois casos obtém "uma tendência semelhante, ou seja, houve redução do índice de Gini e do índice de FosterWolfson $^{6}$, já o índice de DER (Duclos, Esteban, Ray) permaneceu estável, o que demanda novos estudos". A autora parece não perceber que o índice DER mede uma "multipolarização" muito distinta da bipolarização captada pelo índice de FosterWolfson. Ela menciona o artigo de Hoffmann (2008), mas considera que ele trata de "certos efeitos, tais como arredondamentos numéricos quando da declaração pessoal da renda domiciliar, que não serão considerados nesse estudo".

Em outro trabalho de conclusão de curso de graduação orientado pelo Prof. Porto Jr., Schroeder (2014) analisa a polarização da distribuição da renda no Brasil de 2003 a 2012. Comparando a evolução do índice de Gini, da medida de polarização de Foster e Wolfson e do índice DER (Duclos; Esteban; Ray, 2004), ele observa que "apesar de ter tendência declinante, este indicador é muito mais estável que os dois primeiros, indicando que o aumento da classe média teria sido menor se comparado ao outro índice de polarização". Novamente, não percebe que o índice DER mede algo diferente da bipolarização. O artigo de Hoffmann (2008) não é mencionado.

A Figura 6 mostra que a grande frequência de valores iguais ao salário mínimo tem efeito importante sobre a forma da distribuição, como mostrou Soares (2004). Além disso, a tendência de as pessoas informarem sua renda usando números arredondados também afeta a forma da distribuição de maneira relevante. Não se trata de um detalhe desprezível. As linhas verticais na Figura 7 assinalam as abcissas correspondentes a 1/4 do salário mínimo (SM), 1/3 do SM, 1/2 do SM, 1 SM, mil reais $(\mathrm{M})$ e 2 mil reais $(2 \mathrm{M})$. Em todos esses pontos há picos na estimativa da função de densidade obtida usando uma janela relativamente estreita. Como foi mostrado em Hoffmann (2008), a medida de polarização DER reflete a "polarização" nesses picos. Isso não tem nada a ver com a polarização em ricos e pobres e redução de uma classe média. Cabe assinalar que esses picos (especialmente aquele associado ao SM) são ainda mais intensos quando se analisam rendimentos individuais (como renda do trabalho por pessoa ocupada).

(5) No cabeçalho da Tabela 1 do artigo de Hoffmann (2008), onde está $10 P_{E R}$, deve ser $5 P_{E R}$. Evidentemente essa mudança de escala não afeta nenhuma das conclusões do trabalho.

(6) Medida que aqui denominamos índice de Wolfson. 
Figura 6

Três estimativas da função de densidade da distribuição da RDPC em todo o Brasil em 2013: com janela estreita (linha vermelha), com janela 5 vezes mais larga (linha preta) e distribuição log-normal (linha azul claro)

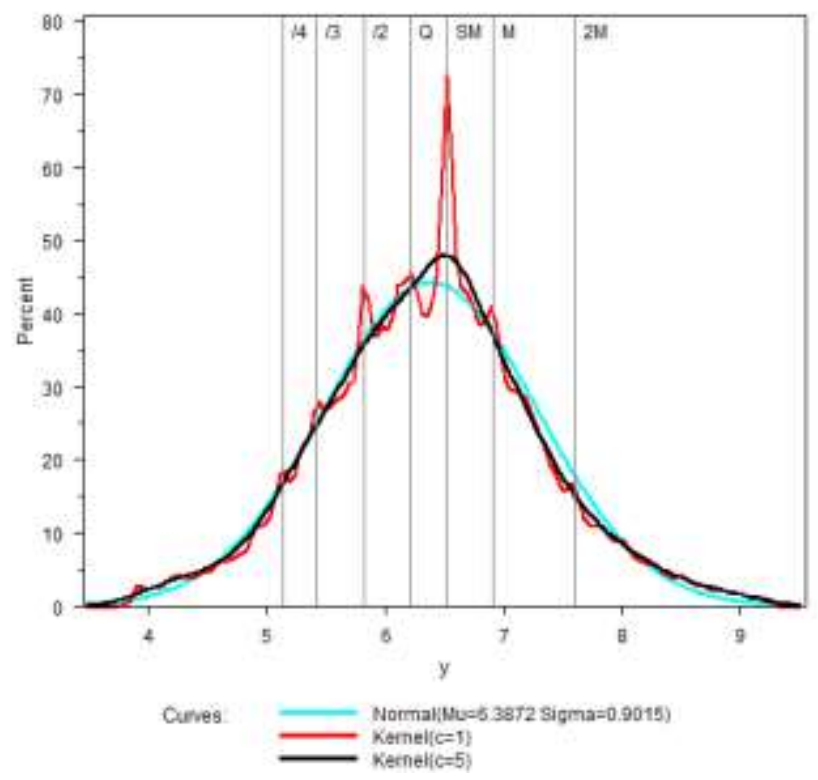

Clementi e Schettino (2013) analisam a distribuição da renda no Brasil de 2001 a 2011 e observam um nítido aumento da polarização de 2004 a 2011. São apresentados vários outros resultados incompatíveis com o que se sabe, com base nos dados da PNAD. Afirmam, por exemplo, que teria ocorrido um forte crescimento da pobreza. Nenhum dos artigos anteriores sobre polarização da distribuição da renda no Brasil é sequer mencionado. $\mathrm{O}$ fato de usarem uma renda por equivalente, dividindo a renda domiciliar pela raiz quadrada do número de pessoas do domicílio, e não a usual renda domiciliar per capita, não é a razão dos resultados discrepantes. Eles calculam o medium relative polarization index (MRP) de Handcock e Morris e fazem o deslocamento da distribuição básica (de 2001) adicionando a diferença entre medianas. Ao comparar a distribuição de 2011 (C) com a de 2001 (A), por exemplo, é criada uma distribuição intermediária $(B)$ adicionando às rendas por equivalente de 2001 cerca de $\mathrm{R} \$ 285$ (com todos os valores em reais de setembro-outubro de 2011), o que é mais do que meio salário mínimo na época. A distribuição $B$ tem desigualdade substancialmente menor do que a da distribuição $A$. Ao comparar a distribuição de 2011 (C) com a distribuição $B$ constata-se um aumento da desigualdade (e um pretenso aumento da polarização), devido à maneira inapropriada de definir a distribuição intermediária. $\mathrm{O}$ artigo deve ser descartado.

Deutsch, Silber e Yalonetzky (2014) analisam a bipolarização de uma medida de nível de vida para 17 países da América Latina. Constatam aumento da bipolarização (de 2000 para 2009) para a maioria dos países, incluindo o Brasil. 
Versões preliminares do trabalho disponíveis na rede permitem verificar que os autores obtiveram, para o Brasil, resultados incompatíveis com a conhecida redução da desigualdade e da pobreza no período. Pelo menos no que se refere aos resultados para o Brasil, esse artigo também não é confiável.

O que foi exposto até esse ponto evidencia que no período de 1995 a 2013, no Brasil, houve redução da polarização da distribuição da renda, acompanhando a redução da desigualdade, e que resultados contrários obtidos por alguns autores se devem a erros metodológicos. Na próxima seção procura-se verificar se a evolução da polarização da distribuição da renda é similar ou não nas diversas regiões do País.

\section{Análise regional}

Nesta seção examina-se a evolução da polarização em cinco regiões: Nordeste, Sudeste, Sul, Centro-Oeste exclusive o Distrito Federal (DF) e o DF. A região Norte é omitida, pois sua comparação com as demais exigiria limitar a análise às áreas urbanas. O DF é destacado tendo em vista as características especiais da remuneração dos funcionários públicos (Daré; Hoffmann, 2013 e Medeiros; Souza, 2012) e o grande peso dessa categoria nessa Unidade da Federação.

A Figura 7 mostra como variou o índice de Gini da RDPC no Brasil e nas 5 regiões. Excetuando-se o caso do DF, é clara a tendência geral de queda da desigualdade.

\section{Figura 7}

Evolução, de 1995 a 2013, do índice de Gini da distribuição da renda domiciliar per capita no Brasil (BR) e em cinco regiões: Nordeste (NE), Sudeste (SE), Sul, Centro-Oeste exclusive DF (CO) e DF

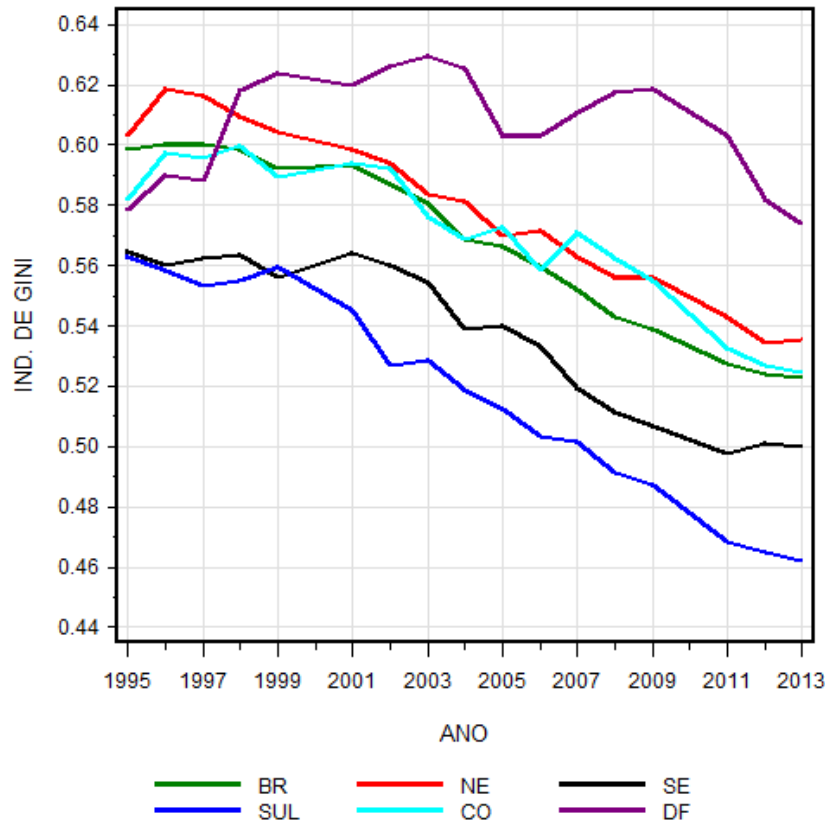


A Figura 8 mostra, para o Brasil (BR) e para as cinco regiões, a evolução de duas medidas de polarização: $P_{W}$ e $P_{M}$. Novamente, excetuando-se o caso do DF, observa-se nítida tendência decrescente.

\section{Figura 8}

Evolução, de 1995 a 2013, de duas medidas de polarização ( $P_{W}$, com linha contínua, e $P_{M}$, com linha tracejada) no Brasil e em cinco regiões: Nordeste, Sudeste, Sul, Centro-Oeste exclusive DF e DF

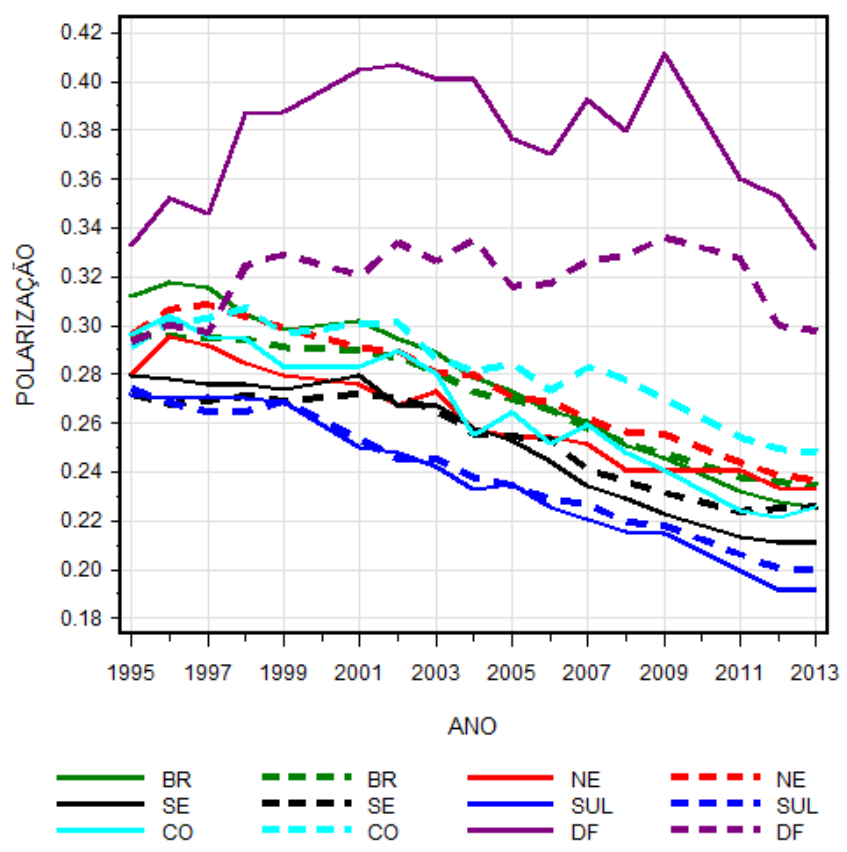

Embora a evolução da polarização da distribuição da RDPC no Distrito Federal seja muito diferente da observada nas demais regiões analisadas, as correlações entre o índice de Gini e as medidas $P_{W}$ e $P_{M}$ para essa Unidade da Federação são fortemente positivas e claramente significativas. De maneira semelhante ao que ocorre nas demais regiões analisadas, no DF a evolução da polarização também tende a acompanhar as mudanças na desigualdade da distribuição da renda.

\section{Conclusão}

Idealmente, a palavra polarização deveria ser reservada para um conceito distinto do de desigualdade, evitando-se usá-la apenas como recurso retórico para ressaltar situações de desigualdade elevada.

Os dados da PNAD permitem constatar que no Brasil, no período 19952013, ocorreu clara redução no grau de bipolarização da distribuição da RDPC, acompanhando a tendência de redução da desigualdade. 
Resultados divergentes apresentados em alguns artigos se devem a uma interpretação errônea de uma medida de multipolarização ou, simplesmente, a erros na análise dos dados.

A tendência de redução da bipolarização também é observada analisando separadamente as regiões Nordeste, Sudeste, Sul e o conjunto dos estados de Mato Grosso do Sul, Mato Grosso e Goiás. Apenas no caso do DF é que se constatou comportamento totalmente distinto, embora também similar à variação da desigualdade da distribuição da RDPC nessa unidade da Federação.

\section{Referências bibliográficas}

AGHEVLI, B. B.; MEHRAN, F. Optimal grouping of income distribution data. Journal of the American Statistical Association, v. 76, n. 373, p. 22-26, mar. 1981.

AITCHISON, J.; BROWN, J. A. C. The lognormal distribution, with special reference to its uses in economics. New York: Cambridge University Press, 1957.

AUDIBERT, P. F. Distribuição e polarização da renda no Rio Grande do Sul: uma evidência empírica. Monografia (Conclusão de Curso)-Faculdade de Ciências Econômicas da UFRGS, 2011.

BARROS, R. P.; FOGUEL, M. N.; ULYSSEA, G. (Org.). Desigualdade de renda no Brasil: uma análise da queda recente. Brasília: Ipea, v. I, 2006 e v. II, 2007.

CASTRO, S. A. C.; SCORZAFAVE, L. G. Ricos? Pobres? Uma análise da polarização da renda para o caso brasileiro. In: ENCONTRO NACIONAL DE ECONOMIA, 33., Anpec, 2005.

CASTRO, S. A. C. Uma análise da polarização de renda no caso brasileiro 1981 2003. Dissertação (Mestrado em Economia)-Universidade Estadual de Maringá, 2006.

CHAKRAVARTY, S. R. Inequality, polarization and poverty. Berlin: Springer, 2009.

CLEMENTI, F.; SCHETTINO, F. Income polarization in Brazil, 2001-2011: a distributional analysis using PNAD data. Economics Bulletin, v. 33, n. 3, p. 1-16, 2013.

CORSEUIL, C. H.; FOGUEL, M. N. Uma sugestão de deflatores para rendas obtidas a partir de algumas pesquisas domiciliares do IBGE. Brasília: Ipea, 2002. (Texto para Discussão, n. 897).

DARÉ, E. F.; HOFFMANN, R. Remuneração do funcionalismo público e a variação da desigualdade da distribuição da renda no Brasil de 1995 a 2009. Economia, v. 14, n. 1c, p. 645-670, 2013. 
DAVIES, J. B.; SHORROCKS, A. F. Optimal grouping of income and wealth data. Journal of Econometrics, v. 42, p. 97-108, 1989.

DEUTSCH, J.; SILBER, J.; YALONETZKY, G. On bi-polarization and the middle class in Latin America: a look at the first decade of the twenty-first century. Review of Income and Wealth, Series 60, Supplement Issue, Nov. 2014.

DUCLOS, J.; ESTEBAN, J.; RAY, D. Polarization: concepts, measurement, estimation. Econometrica, v. 72, n. 6, p. 1737-1772, 2004.

ESTEBAN, J.; GRADÍN, C.; RAY, D. An extension of a measure of polarization, with an application to the income distribution of five OECD countries. Journal of Economic Inequality, v. 5, p. 1-29, 2007.

ESTEBAN, J.; RAY, D. On the measurement of polarization. Econometrica, v. 62, n. 4, p. 819-851, 1994.

FIGUEIRÊDO, E. A.; SILVA NETTO JR., J. L.; PORTO JR., S. Distribuição, mobilidade e polarização de renda no Brasil: 1987 a 2003. Revista Brasileira de Economia, v. 61, n. 1, p. 7-32, 2007.

FOSTER, J. E.; WOLFSON, M. C. [Jul. 1992]. Polarization and the decline of the middle class: Canada and the US. Oxford: Oxford Poverty \& Human Development Initiative, Aug. 2009. (OPHI Working Paper, n. 31).

HANDCOCK, M. S.; MORRIS, M. Relative distribution methods in the social sciences. New York: Springer, 1999.

HOFFMANN, R. Effect of the rise of a person's income on inequality. Brazilian Review of Econometrics, v. 21, n. 2, p. 237-262, Nov. 2001.

HOFFMANN, R. Desigualdade de renda no Brasil em 2005 e sua decomposição. Capítulo do CD "Análise da Pesquisa Nacional por Amostra de domicílios - PNAD 2005", Livro 3, "Pobreza e Desigualdade". Brasília: Centro de Gestão e Estudos Estratégicos - CGEE, 2007. Disponível em: www.cgee.org.br/.

HOFFMANN, R. Polarização da distribuição da renda no Brasil. Econômica, v. 10, n. 2, p. 169-186, dez. 2008.

LAMBERT, P. J.; LANZA, G. The effect on inequality of changing one or two incomes. The Journal of Economic Inequality, v. 4, p. 253-277, 2006.

LOPES, E. S. Desigualdade e polarização da distribuição de renda no Brasil e no Estado de São Paulo, 1992-2007. Dissertação (Mestrado)-Unicamp, Instituto de Economia, 2009.

MEDEIROS, M.; SOUZA, P. H. G. F. Gasto público, tributos e desigualdade de renda no Brasil. Brasília: Escola de Administração Fazendária (ESAF) e Tesouro Nacional, 2012. (XVII Prêmio Tesouro Nacional-2012). 
MILANOVIC, B. A new polarization measure and some applications. Washington, DC: World Bank. Development Research, 2000.

SCHROEDER, G. N. Polarização de renda no Brasil: uma análise por macrorregião. Monografia (Conclusão de Curso)-Faculdade de Ciências Econômicas da UFRGS, 2014.

SCORZAFAVE, L. G.; CASTRO, S. A. C. Ricos? Pobres? Uma análise da polarização da renda para o Brasil - 1981-2003. Pesquisa e Planejamento Econômico, v. 37, n. 2, p. 283-297, 2007.

SOARES, S. S. D. O impacto distributivo do salário mínimo: a distribuição individual dos rendimentos do trabalho. Economia Aplicada, v. 8, n. 1, p. 47-76, 2004.

WOLFSON, M. C. When inequalities diverge. American Economic Review, v. 84, n. 2, p. 353-358, 1994.

WOLFSON, M. C. Divergent inequalities: theory and empirical results. Review of Income and Wealth, v. 43, n. 4, p. 401-421, 1997. 